\title{
Choice of Management in a Rare Case of Symptomatic Wolff-Parkinson-White (WPW) Syndrome Type B
}

\author{
Choe Yee Xian', Rafiq Shajahan'1, Puay Sheng Hwa1, Naganathan Pillai', \\ Ganesh Kasinathan ${ }^{2}$ \\ ${ }^{1}$ Department of Medicine, Monash University Malaysia, Subang Jaya, Malaysia \\ ${ }^{2}$ Department of Medicine, Segamat Hospital, Segamat, Johor, Malaysia \\ Email: yxchoe@gmail.com, concorde842000@yahoo.com
}

Received 24 November 2015; accepted 10 December 2015; published 14 December 2015

Copyright (C) 2015 by authors and OALib.

This work is licensed under the Creative Commons Attribution International License (CC BY).

http://creativecommons.org/licenses/by/4.0/

(c) (i) Open Access

\begin{abstract}
Introduction: Wolff-Parkinson-White (WPW) syndrome type B is a congenital condition involving abnormal electrical conduction between the atria and the ventricles that provide an accessory pathway (AP) for a re-entrant tachycardia circuit. Background: This case report illustrates a 28year-old male who presented with multiple episodes of rapid, regular palpitations associated with dizziness and nausea. These episodes of palpitations often resolve spontaneously. Physical examination revealed normal first and second heart sounds with no audible murmurs. Other systemic examinations were unremarkable. A 12 lead electrocardiogram showed an atrioventricular re-entrant tachycardia (AVRT) of 210 beats per minute. Intravenous amiodarone was given to which he responded. Post pharmacological cardioversion, the repeated ECG showed shortened PR interval and broad QRS complexes associated with negative delta waves in lead V1, T-wave inversion in inferior leads and precordial leads of V5-V6 which represented a Type B pattern. He was then referred to the electrophysiology unit at the National Heart Institute for radiofrequency ablation (RFA) and Holter monitoring. Conclusion: Wolff-Parkinson-White (WPW) Type B should be considered as a differential diagnosis for a young individual who presents with frequent palpitations. RFA is well known as a permanent solution to the prevention of tachyarrhythmia.
\end{abstract}

\section{Keywords}

Wolff-Parkinson-White Type B, Accessory Pathway, Amiodarone, Palpitation, Radiofrequency Ablation

Subject Areas: Cardiology

How to cite this paper: Xian, C.Y., Shajahan, R., Hwa, P.S., Pillai, N. and Kasinathan, G. (2015) Choice of Management in a Rare Case of Symptomatic Wolff-Parkinson-White (WPW) Syndrome Type B. Open Access Library Journal, 2: e2219. 


\section{Introduction}

Wolff-Parkinson-White (WPW) syndrome represents one of many conditions with paroxysms of tachyarrhythmias that belong to a unifying pathophysiology of pre-excitation [1]. Specifically, there is a congenital embryological formation of accessory conductive pathway that connects the atrium and ventricle that bypasses the atrioventricular node. Hence, the presence of two atrioventricular connections and differing refractory periods between them can eventually lead to unique supraventricular tachycardia called an atrioventricular re-entrant tachycardia (AVRT) [1]-[3]. Estimates of WPW syndrome prevalence range from $0.068 \%$ to $0.174 \%$, with WPW syndrome being twice as common in those with coronary heart disease [4] [5]. WPW syndrome is divided into type A and Type B with unknown measures of prevalence in the population. However it is thought that Type A is more common as left sided accessory pathways (APs) are much more common than right APs as visualized by electrophysiological studies. All WPW syndromes have a small risk of sudden cardiac death with an incidence of 1 to 1000 patient-years in those with presence of ventricular pre-excitation [3]. There are also two other pre-excitation syndromes, namely Lown-Ganong-Levine syndrome and Mahaim types [1] [2].

\section{Case Presentation}

A 28-year-old Malay gentleman presented with multiple episodes of palpitations for the past one year in which he described the palpitations as rapid and regular. The palpitation was associated with dizziness, nausea and dyspnoea. He denied any syncope or chest pain. There were no symptoms of heart failure. He had no family history of sudden cardiac death (SCD) or palpitations. He denied any use of over the counter, herbal, traditional, stimulant and recreational drug use. He almost never consumed any caffeinated products. He is a non-smoker and a teetotaler. He works at a textile factory. Physical examination revealed an alert male who was clinically pink with no jaundice. He had no obvious goiter. He was afebrile with a blood pressure of 139/76 mmhg. The heart rate was 210 beats per minute regularly regular. Oxygen saturation was $100 \%$ on room air. Bedside capillary glucose was $5.2 \mathrm{mmol} / \mathrm{L}$. The cardiovascular examination revealed first and second heart sounds with no audible murmurs. Jugular venous pulsations were not elevated and the apex beat was not displaced. Lungs were clear on auscultation. Other systemic examinations were unremarkable. There was no lower limb edema. The 12 lead electrocardiogram showed atrioventricular re-entrant tachycardia (AVRT) of 210 beats per minute. The complete blood count, renal profile, liver function test, electrolytes and thyroid function test are as shown in Table 1. The patient was given intravenous amiodarone $150 \mathrm{mg}$ which was diluted in $100 \mathrm{ml}$ of Dextrose $5 \%$ solution over 15 minutes followed by $360 \mathrm{mg}$ of amiodarone over 8 hours and then $540 \mathrm{mg}$ over 16 hours. The

Table 1. Blood parameters of the patient.
\begin{tabular}{|cc|}
\hline Parameters (units) & Values (normal range) \\
\hline Haemoglobin $(\mathrm{g} / \mathrm{dL})$ & $13.8(13-18)$ \\
White cell count $\left(10^{9} \mathrm{~L}\right)$ & $9.5(4-11)$ \\
Platelet $\left(10^{9} \mathrm{~L}\right)$ & $253(150-450)$ \\
Albumin $(\mathrm{g} / \mathrm{L})$ & $40(35-55)$ \\
AST $(\mathrm{U} / \mathrm{L})$ & $30(10-40)$ \\
ALT $(\mathrm{U} / \mathrm{L})$ & $25(10-40)$ \\
Creatinine kinase $(\mathrm{U} / \mathrm{L})$ & $153(52-336)$ \\
Sodium $(\mathrm{mmol} / \mathrm{l})$ & $140(136-145)$ \\
Potassium $(\mathrm{mmol} / \mathrm{l})$ & $4(3.5-5)$ \\
Serum Creatinine $(\mu \mathrm{mol} / \mathrm{l})$ & $78(60-110)$ \\
Calcium $(\mathrm{mmol} / \mathrm{l})$ & $2.5(2.2-2.6)$ \\
Magnesium $(\mathrm{mmol} / \mathrm{l})$ & $0.9(0.7-1.1)$ \\
Phosphate $(\mathrm{mmol} / \mathrm{l})$ & $0.9(0.8-1.5)$ \\
\hline
\end{tabular}


12 lead electrocardiogram post pharmacological cardioversion showed shortened PR interval, slurred downstroke of the QRS complex in V1, broad QRS complex with T-wave inversion in V5, V6 and inferior leads as shown in Figure 1. A 2-dimensional transthoracic echocardiogram showed no structural abnormality with good left ventricular ejection fraction of $65 \%$ and no left ventricular hypertrophy. Chest radiograph showed clear lung fields with a normal cardiac size. A diagnosis of WPW syndrome type B was made. Patient was then referred for RFA and Holter monitoring at the National Heart Institute.

\section{Discussion}

Wolff-Parkinson-White (WPW) is a syndrome that presents with paroxysms of tachycardia that have a distinctive pathological feature, namely pre-excitation [1]-[3]. It requires 1) an alternate pathway of conduction that connects the atrium and ventricle (Bundle of Kent) and 2) differing electrical characteristics of these two pathways that predispose it to a re-entrant tachyarrhythmia [1]. Current electrophysiological evidence supports the theory advanced first by de Boer in 1926 that describes a circus-like movement in electrical conductance of the heart consisting of an impulse from the atrium to atrioventricular junction followed by the His-bundle towards the ventricle and finally back up to the atrium via $\mathrm{AV}$ anomalous conduction (later known as an Orthodromic AVRT) [6]. Often, when this occurs, patients may present to the hospital with bouts of palpitations with the characteristic resemblance of a supraventricular tachycardia (SVT) on a standard 12-lead electrocardiogram. Symptoms are variable with differing severity related to the meaningful cardiac output such as palpitations, syncope, chest pain and dyspnea [1] [3]. When the AVRT ceases, the patient would return to the premorbid state of health before the next attack occurs. These pathways usually exhibit separate conductive properties specifically in their refractory periods and/or speeds [1] [3]. In this particular scenario, the properties of the AP in WPW syndrome are as follows [1]:

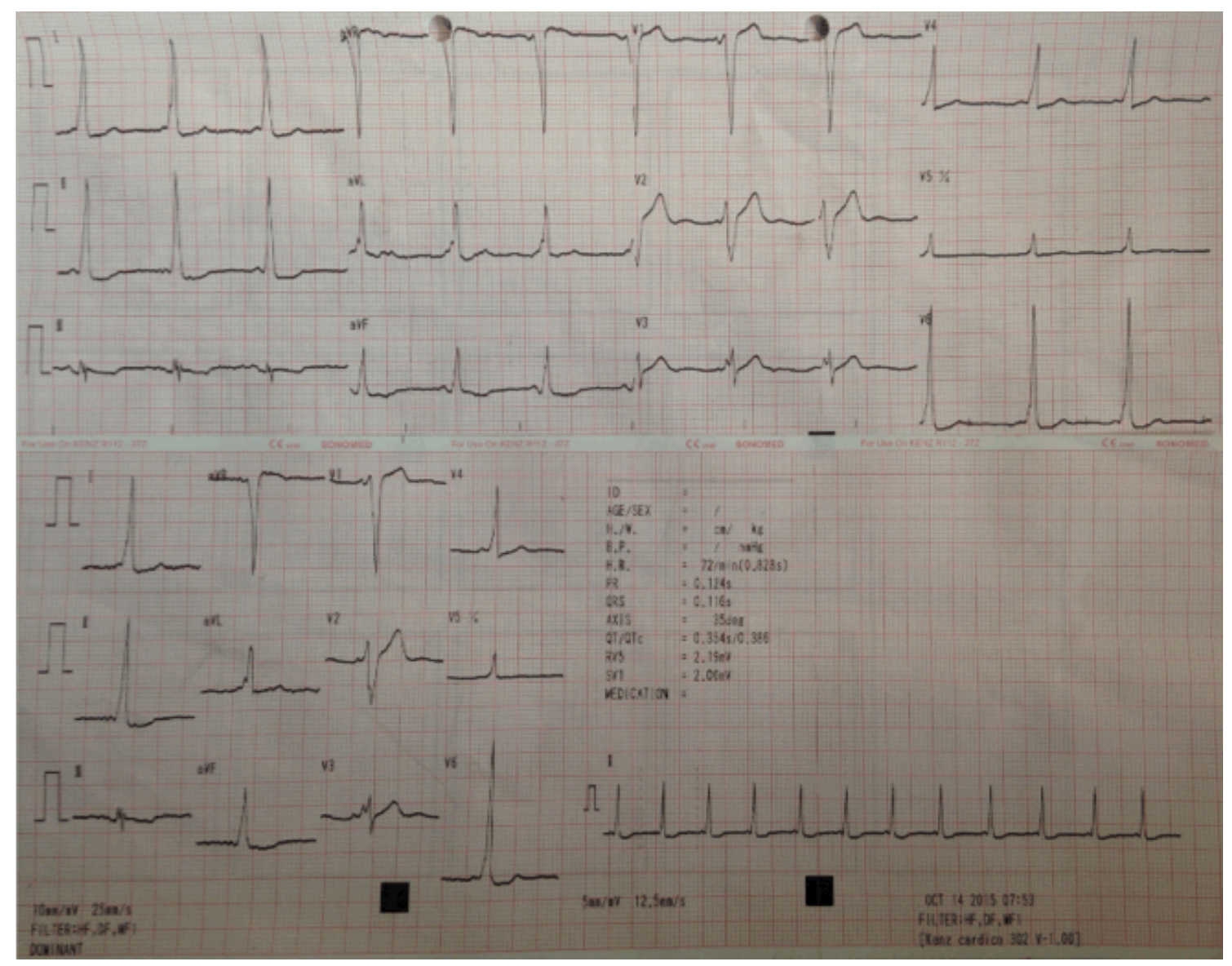

Figure 1. 12 lead electrocardiogram of patient. 
1) Faster conduction speeds than the AV-His bundle (as the AV node has a normal delay to allow ventricular filling.

2) Longer refractory period than the AV-His bundle

This leads to the faster AP being blocked due to its own extended refractory time allowing the impulse in the AV-His bundle pathway to continue retrograde up the AP, predisposing a re-entrant tachycardia [1]. Re-entrant type tachycardia can occur either due to APs or even presence of ischemic tissue [1]. A firm grasp of the basis of AVRT underscores the ultimate aim of medical cardioversion in which slowing conduction speeds and prolonging refractory periods in the AP, to allow more AV conductance is the desired outcome [3]. Following this revelation, AVRTs can be precipitated by any AV nodal blocking drugs such as Adenosine, Verapamil, or Digitalis and many well-revised guidelines have recommended avoiding these classes of drugs instead suggesting Class 1a, 1c and 3 anti-arrhythmic medications in its place [2]. The overwhelming majority of ECG patterns in sinus rhythm follow the type A pattern with a smaller incidence of type B patterns. The ECG findings between type A and type B are as tabulated in Table 2 [2]. These different types merely signify the site of the AP with Type A patterns occurring due to left sided APs and Type B patterns occurring due to right-sided APs [7]. This finding has been reinforced with evidence from various sources such as when Durrer and Roos were the very first ones to locate a right free wall AP via intraoperative mapping and cooling [7]. Since the advent of radiofrequency ablation as a suitable method to successfully treat this condition, algorithms have been developed to accurately predict APs in ECGs for multiple reasons; to reduce morbidity, shorten procedure time, selection of the ablator with appropriate curves and to minimize mechanical trauma [8] [9]. One of the first known attempts was in an article written in 1987 by Milsten and Sharma et al. published an algorithm by attempting to identify four APs in 141 patients, namely right free wall, anteroseptal, posteroseptal and left free wall using the morphology of delta wave [8]. Following that, Chiang and Chen et al in 1995 began to embark on the same endpoint by including the R/S ratio of the QRS complex along with the Delta wave morphology in 369 patients with only anterograde aberrant pathways. They did this by first examining 182 patients' pre-ablation ECGs and electrophysiology findings, noting the polarity of delta waves of the earliest pre-excitations among the precordial leads. They then went on to develop a relatively simple 4-step process that required examining 4 leads: V1, V2, Lead III and aVF. They discovered that their algorithm was able to predict the location of APs with an overall accuracy of 93\% when they used the algorithm to predict their remaining 187 patients as compared to $86 \%$, 85\% and $85 \%$ respectively [9]. In 2014, a paper by Taguchi and Yoshida et al recognized that using the delta wave morphology analysis method can often be difficult with many limitations such as the need for maximal pre-excitation. They proposed amendments to just use the R/S ratio to accurately predict the location of these APs. Using 142 patients (88 post ablative ECGs of patients to construct algorithm and 54 patients for prospective testing of developed algorithm) they were able to just use only the R/S ratios with assessments of V1, V2 and aVF to correctly identify the correct aberrant pathway 51 out of 54 patients. This translated to an overall sensitivity of $94 \%$ and specificity of $98 \%$ [10]. Since its introduction in 1987, the utilization of RFA has now been recognized as a treatment of choice for WPW with tachyarrhythmias due to its relative safety and efficacy, as well as for the primary rationale of preventing the occurrence of sudden cardiac deaths [4] [11]. Electrophysiology studies, which involve the placement of electrode catheters within the cardiac chambers, enable inter-cardiac signals to be recorded in real-time and are coupled with RFA procedures to map out the APs that need to be ablated [12]. The overall success rate of RFA is approximately 93\%, of which ablation of left free wall APs have a success rate of 95\%, followed by right free wall (90\%) and posteroseptal APs (88\%) [4]. This is in part due to the difficulty

\section{Table 2. ECG findings of WPW type A and Type B.}

\begin{tabular}{|c|c|}
\hline Type A findings & Type B Findings \\
\hline Short PR interval ( $<120 \mathrm{~ms}$ ) waves in sinus rhythm & Short PR interval ( $<120 \mathrm{~ms}$ ) waves in sinus rhythm \\
\hline $\begin{array}{c}\text { Presence of Broad QRS complex accompanied } \\
\text { with slurred upstroke R component which } \\
\text { could be a possible RBBB mimicker (Delta wave) }\end{array}$ & $\begin{array}{l}\text { Presence of Broad QRS complex accompanied } \\
\text { with slurred downstroke S component which } \\
\text { could a possible LBBB mimicker (Delta wave) }\end{array}$ \\
\hline Dominant R wave In V1 (Left sided AP) & Dominant S wave in V1 (right sided AP) \\
\hline $\begin{array}{c}\text { Tall R waves accompanied with inverted T } \\
\text { waves in leads V1 until V3 (mimicker of RVH) }\end{array}$ & $\begin{array}{l}\text { Tall R waves accompanied with inverted T waves in the } \\
\text { inferior leads and V4 till V6 (mimicker of LVH) }\end{array}$ \\
\hline
\end{tabular}


in ablating the latter two APs, which is also reflected in the high proportion of second ablation procedures required. In addition, high volume ablation centers also concur with relatively higher rates of success [13]. A recent epidemiological study in Taiwan suggests that the implementation of RFA in its health system has contributed to a lower prevalence rate of WPW compared to prior population studies, with an overall prevalence of (0.36/1000) and a peak prevalence rate of 0.61/1000 [4]. Current debate revolves around the appropriate clinical approach to asymptomatic WPW patients and the need to strike a balancing act between taking necessary measures to prevent sudden cardiac death while avoiding the potential complications brought about by the invasive nature of RFA. In an observational study spanning 8 years by Pappone et al. on 2169 patients, it was found that prophylactic RFA was able to reduce the frequency of arrhythmias in patients diagnosed with WPW syndrome [14].

\section{Conclusion}

WPW type B should always be considered as an important differential diagnosis in a young male who presents with frequent palpitations. It causes for sudden cardiac death. Hence a high index of suspicion coupled with a good clinical workup and management may help prevent morbidity and mortality associated with this disorder. RFA remains the mainstay modality of treatment with regards to a permanent cessation of its associated tachyarrhythmias.

\section{Acknowledgements}

We would like to acknowledge the contributions of the Head of Department of Medicine, Segamat Hospital, Dr. R. Sirajudeen for his motivation and guidance throughout the production of our case study.

\section{Informed Consent}

Informed consent was obtained from the patient for the publication of this study.

\section{References}

[1] Kulig, J. and Koplan, B.A. (2010) Wolff-Parkinson-White Syndrome and Accessory Pathways. Circulation, 122, 480483. http://dx.doi.org/10.1161/CIRCULATIONAHA.109.929372

[2] Keating, L., Morris, F.P. and Brady, W.J. (2003) Electrocardiographic Features of Wolff-Parkinson-White Syndrome. Emergency Medicine Journal, 20, 491-493. http://dx.doi.org/10.1136/emj.20.5.491

[3] Mark, D., Brady, W. and Pines, J. (2009) Preexcitation Syndromes: Diagnostic Consideration in the ED. The American Journal of Emergency Medicine, 27, 878-888. http://dx.doi.org/10.1016/j.ajem.2008.06.013

[4] Lu, C., Wu, M., Chen, H., Kao, F. and Huang, S. (2014) Epidemiological Profile of Wolff-Parkinson-White Syndrome in a General Population Younger than 50 Years of Age in an Era of Radiofrequency Catheter Ablation. International Journal of Cardiology, 174, 530-534. http://dx.doi.org/10.1016/j.ijcard.2014.04.134

[5] De Bacquer, D. (2000) Prevalences of ECG Findings in Large Population Based Samples of Men and Women. Heart, 84, 625-633. http://dx.doi.org/10.1136/heart.84.6.625

[6] Deboer, S. (1926) Die physiologiche Grundlage und Klinik des unregelmassigen Herzschlages. Ergebnisse der Inneren Medizin und Kinderheilkunde, 29, 391-514.

[7] Durrer, D. and Roos, J. (1967) Epicardial Excitation of the Ventricles in a Patient with Wolff-Parkinson-White Syndrome (Type B). Circulation, 35, 15-21. http://dx.doi.org/10.1161/01.CIR.35.1.15

[8] Milsten, S., Sharma, A.D., Guiraudon, G.M., et al. (1987) An Algorithm for the Electrocardiographic Localization of Accessory Pathways in the Wolff-Parkinson-White Syndrome. Cardiac Investigational Unit of University Hospital, London, Ontario, Canada, Vol. 10, 555-565.

[9] Chiang, C.E., Chen, S.A., Teo, W.S., et al. (1995) An Accurate Stepwise Electrocardiographic Algorithm for localization of Accessory Pathways in Patients With Wolff-Parkinson-White Syndrome from a Comprehensive Analysis of Delta Waves and R/S Ratio During Sinus Rhythm. The American Journal of Cardiology, 76, 40-46. http://dx.doi.org/10.1016/S0002-9149(99)80798-X

[10] Taguchi, N., Yoshida, N., Inden, Y., Yamamoto, T., Miyata, S., Fujita, M., et al. (2014) A Simple Algorithm for Localizing Accessory Pathways in Patients with Wolff-Parkinson-White Syndrome Using Only the R/S Ratio. Journal of Arrhythmia, 30, 439-443. http://dx.doi.org/10.1016/j.joa.2013.10.006 
[11] Svendsen, J., Dagres, N., Dobreanu, D., Bongiorni, M., Marinskis, G. and Blomstrom-Lundqvist, C. (2013) Current Strategy for Treatment of Patients with Wolff-Parkinson-White Syndrome and Asymptomatic Preexcitation in Europe: European Heart Rhythm Association survey. Europace, 5, 750-753. http://dx.doi.org/10.1093/europace/eut094

[12] American College of Cardiology/American Heart Association (2006) Update of the Clinical Competence Statement on Invasive Electrophysiology Studies, Catheter Ablation and Cardioversion: A Report of the American College of Cardiology/American Heart Association/American College of Physicians Task Force on Clinical Competence and Training: Developed in Collaboration With the Heart Rhythm Society. Circulation, 114, 1654-1668.

[13] Calkins, H., Yong, P., Miller, J., Olshansky, B., Carlson, M., Saul, J., et al. (1999) Catheter Ablation of Accessory Pathways, Atrioventricular Nodal Reentrant Tachycardia, and the Atrioventricular Junction: Final Results of a Prospective, Multicenter Clinical Trial. Circulation, 99, 262-270. http://dx.doi.org/10.1161/01.CIR.99.2.262

[14] Pappone, C., Vicedomini, G., Manguso, F., Saviano, M., Baldi, M., Pappone, A., et al. (2014) Wolff-Parkinson-White Syndrome in the Era of Catheter Ablation: Insights from a Registry Study of 2169 Patients. Circulation, 130, 811-819. http://dx.doi.org/10.1161/CIRCULATIONAHA.114.011154 